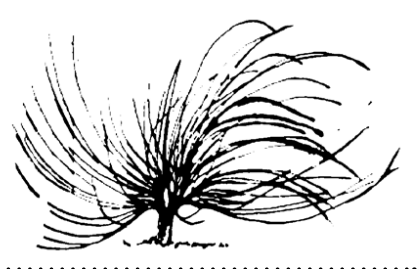

\title{
Evaluación de la conferencia académica como herramienta de desarrollo profesional y de fortalecimiento de capacidades en profesores de idiomas
}

\author{
Lena Barrantes Elizondo ${ }^{1}$ \\ Universidad Nacional \\ Costa Rica \\ lenna.barrantes.elizondo@una.ac.cr \\ Cinthya Olivares Garita ${ }^{2}$ \\ Universidad Nacional \\ Costa Rica \\ coliga@una.ac.cr
}

\begin{abstract}
Resumen
Las conferencias poseen un carácter divulgativo del quehacer académico, que las ha convertido en un discurso habitual en el círculo académico. Este tipo de actividad favorecedora del desarrollo profesional ha llegado a adquirir cierta obligatoriedad en el ámbito universitario. Es así como se entiende que las conferencias académicas son, para la educación universitaria, una forma comunicativo-cognitiva imprescindible. Este trabajo investigativo realiza la evaluación de una conferencia académica,
\end{abstract}

Recibido: 9 de junio de 2020. Aprobado: 1 de junio de 2021.

http://dx.doi.org/10.15359/rep.16-2.11

1 Doctorado en Filosofía ( $\mathrm{Ph}$. D), en Investigación Educativa con énfasis en Aprendizaje de Adultos, de la Universidad de Calgary. Maestría en Segundas Lenguas y Culturas con énfasis en Inglés como Lengua Extranjera para Alumnado Adulto y Maestría en Gestión Educativa con énfasis en Liderazgo, de la Universidad Nacional de Costa Rica. Actualmente, profesor en la Universidad Nacional, Sede Regional Brunca. ORCID: https://orcid. org/0000-0003-3242-226X

2 Magíster en Segundas Lenguas y Culturas con énfasis en Inglés como Lengua Extranjera para Alumnado Adulto y Magíster en Gestión Educativa con énfasis en Liderazgo, de la Universidad Nacional de Costa Rica. En la actualidad, docente en la Universidad Nacional, Sede Regional Brunca. ORCID: https://orcid.org/0000-0001-7516-2715 
según principios de la teoría de evaluación de programas. Dicha valoración siguió tres enfoques de evaluación orientada: a programas, a la toma de decisiones y a los participantes. A pesar de que impacta un contexto específico, sus resultados devienen en prácticas que benefician la implementación de la conferencia académica como herramienta de desarrollo profesional docente. Se concluyó que el papel emancipador que otorga la universidad a través de la difusión de conocimiento es vital para acompañar a docentes de inglés, en tanto se fortalezca su lente colectivo, en procura de una mayor interacción social. Las redes de relaciones colaborativas, como las conferencias académicas, promueven la retroacción y la humanización de los vínculos sociales del docente, quien aprende en colaboración con otros.

Palabras clave: Conferencia académica, desarrollo profesional, evaluación de programas

\begin{abstract}
Conferences possess an informative nature about the academic work that recognizes them as a regular practice within the professional academic arena. This type of activity that favors teachers' professional development has become compulsory at the university level through time. In this way, academic conferences are an essential communicative-cognitive source for higher education. This study encompasses the evaluation of an academic conference by following principles of program evaluation. That valuation followed three approaches: program-oriented evaluation, decision-oriented evaluation, and participant-oriented evaluation. Although it impacts a project of a specific context, its results inform successful practices that might benefit similar initiatives. The dissemination of these data constitutes input to make thought-out and assertive decisions for the execution of effective and enriching academic conferences that would promote teachers' professional development. It was concluded that the emancipatory role that the university grants to the individual through the spreading of knowledge is vital for the accompaniment of teachers of English inasmuch as their collective lens is strengthened so that greater social
\end{abstract}


interaction is achieved. Collaborative networks, as constituted through academic conferences, promote feedback and humanization of teachers' social relations who learn in collaboration with others.

Keywords: academic conference, professional development, program evaluation

\section{Introducción}

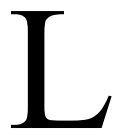

a virtud más significativa de las conferencias académicas

o especializadas yace en el valor humano que conlleva el establecimiento de redes sociales con profesionales de la misma área de especialización. Algren et al. (2011) lo mencionan como el elemento "humanidad". Las conferencias, como espacio que favorece el desarrollo profesional, supera a mecanismos impresos de conocimiento (libros y revistas académicas), al proveer oportunidades para ampliar redes de contactos y participación en conversaciones académicas. Estos recursos poseen un carácter divulgativo del quehacer académico que los ha convertido en un discurso habitual en ese círculo del saber. Tal tipo de desarrollo profesional ha llegado a adquirir cierta obligatoriedad en el ámbito universitario. Es así como se entiende que las conferencias académicas son, para la educación universitaria, una forma comunicativo-cognitiva imprescindible (Sorokina, 2015). Señalan Ruíz-Díaz et al. (2017) que

la conferencia especializada permite dar una visión panorámica del estado del arte de las áreas temáticas fundamentales, familiariza a sus participantes con esos contenidos ... [, lo que a la vez permite al docente] ... destacar lo esencial ante la gran cantidad de materiales y flujo de información al respecto. (p. 14)

El objetivo del estudio fue el análisis de la evaluación del proyecto Conferencia Internacional de Lingüistica Aplicada (CONLA UNA), basado en los principios de valoración sugeridos por Fitzpatrick et al. (2011). Este análisis se realizó con el fin de determinar el impacto de la conferencia académica como herramienta de desarrollo profesional en una universidad en Costa Rica. Para esto, se siguieron tres enfoques sugeridos por los autores antes mencionados: evaluación orientada a 
programas, evaluación orientada a la toma de decisiones y evaluación orientada a los participantes.

El posicionamiento epistemológico que esta investigación tomó se basa en las ideas del paradigma participativo posmodernista expuesto por Lincoln et al. (2018), el cual establece como determinante el conocimiento práctico y la subjetividad crítica. Los criterios de calidad miden la congruencia vivencial y el saber práctico que conlleva acciones al servicio del florecimiento y de la transformación del ser humano, en este caso, al mejoramiento de procesos educativos. En el paradigma aludido, las voces principales se manifiestan a través de la acción-reflexión y la interpretación holística. Se hace también referencia a principios ontológicos, en los que la realidad participativa se coconstruye entre la mente y el cosmos circundante (Guba y Lincoln, 2005), situación que genera una construcción social. Es así como la base del conocimiento es construida por y para los actores. Ese paradigma se alinea con principios de valoración de proyectos o programas como estrategia de mejoramiento de la educación superior. De manera específica, la evaluación de programas es un método sistemático para recopilar, analizar y utilizar información, con miras a responder preguntas sobre la efectividad y eficacia de proyectos, políticas y programas educativos. Este método permite desarrollar estrategias de espacios de reflexión crítica y análisis social que competen e involucran las voces de los estudiantes, docentes, personal administrativo e integrantes de la comunidad. La evaluación de proyectos da pie al elemento transformativo institucional, al proveer dirección e insumos para la toma de decisiones futuras, fundamentado en la acción-reflexión.

\section{Referentes teóricos}

\section{Mecanismos de desarrollo profesional y formación docente}

El término desarrollo profesional ha traído a la mesa de discusión de la comunidad científica y docente diferentes puntos de vista acerca de su conceptualización. Ha tomado relevancia en los últimos años, pero su vínculo con la formación del profesorado se remonta a varias décadas anteriores. Resulta difícil consensuar su definición, sin embargo, Imbernón y Canto (2013) alertan que el desarrollo profesional no es simplemente un conjunto de prácticas o acciones para la mejora 
de competencias, actitudes o el cumplimiento de una función determinada. Esto reduciría el concepto a un simplismo funcionalista.

Según los autores, la formación permanente se nutre del "desarrollo pedagógico, al conocimiento y comprensión de sí mismo, al desarrollo cognitivo o al teórico" (Imbernón y Canto, 2013, p. 3), pero el desarrollo profesional, como término general, engloba la formación profesional sumada a los factores de índole institucional, laboral, salarial y de profesionalización. Dicho de otro modo, este tipo de desarrollo, encausado a mejorar la calidad docente, investigativa y de gestión, implica toda acción sistemática realizada en beneficio de la práctica laboral y del mejoramiento de actitudes, competencias y conocimientos profesionales (Imbernón y Canto, 2013). Desde esta perspectiva, CONLA UNA es un mecanismo que favorece, ciertamente, el desarrollo profesional de los docentes, ya que sus fines se orientan hacia el fortalecimiento de las competencias del profesorado de inglés, al mismo tiempo que brinda la oportunidad de ascender de categoría o, en su condición laboral, en plazas fijas o permanentes.

El ser humano, como ente social, requiere el contacto y apoyo de otras personas para satisfacer sus necesidades de comunicación y superación. De la misma forma, los docentes necesitan de sus pares para progresar en su desarrollo educativo y así realizarse en su dimensión de ser-con-otros, como seres sociales, sin limitarse a una simple reacción. Este ser-con-otros se deriva de la idea heideggeriana de la disposición de ayudar a los demás y tender la mano. Ello abre espacios para construir e integrar comunidad.

Pertenecer a un grupo social determinado favorece que el ser humano construya su propia identidad y contemple su papel como individuo, al formar parte de un grupo de iguales que comparten miedos, triunfos, frustraciones y metas. Este sentido de pertenencia conlleva que los docentes tengan claro su rol protagónico en su propio espacio de trabajo y su función como integrante de un equipo de apoyo del cual se tienen expectativas.

Asimismo, Wolfram y Schilling-Estes (2006) explican: "se entiende que la identidad incluye dimensiones personales e interpersonales, ya que las personas se definen a sí mismas en relación con otros y deciden proyectar diferentes aspectos de nuestra identidad a diferentes tipos de audiencia (p. 287)." El que los docentes puedan definirse e identificarse en dimensiones profesionales puede contribuir a 
que asuman un alto sentido de responsabilidad social, el cual los lleva a querer fortalecer sus habilidades y destrezas personales.

Para este propósito, el desarrollo implica un claro compromiso docente que manifieste otro personal y social. La idea de que esta es una extensión a la carga horaria y una obligación más impide su pleno aprovechamiento. Existe una evidente necesidad de que sea el mismo maestro quien propicie y anhele ese espacio de formación. La responsabilidad personal de los docentes requiere que estos sean capaces de identificar su justo valor y anhelen asumir su papel protagónico en la sociedad. De igual manera, es preciso que los educadores asuman sus deberes y establezcan objetivos, como el aspirar ser profesionales habilitados para reflexionar, evaluar, replantear y cambiar su propia práctica docente, para responder a las necesidades educativas vigentes en su contexto social.

Un elemento clave para el desarrollo de una sociedad es un sistema educativo que no solo satisfaga requerimientos específicos, sino que pueda predecir futuros cambios, los cuales le den a su ciudadanía las herramientas para afrontar retos de corto y largo plazo. Es aquí donde el papel del profesor toma relevancia: observar, de manera tácita, las realidades inmediatas y tomar una posición de mediador entre las demandas curriculares y estas realidades. El compromiso social del educador yace en la ejecución de un análisis de introspección que le permita ver la importancia de su rol como integrante del grupo social que lo rodea y de cuán indispensable es su labor como sujeto en la producción del saber de esta comunidad específica.

\section{Desarrollo profesional de docentes de inglés}

El profesorado de inglés tiene una triple tarea en su desarrollo profesional: velar por la actualización sobre prácticas metodológicas efectivas, exitosas y novedosas; además, pulir e incrementar su capacidad en el manejo apropiado del idioma. Además, como un valor agregado en su formación, debe fortalecer la nueva identidad vinculada al uso de esta segunda lengua, como medio de construcción y expresión de su propia identidad. Bajo este entendido, todo mecanismo de desarrollo profesional requiere la integración de los ejes pedagógico y didáctico-disciplinar de manera interdisciplinaria (Chacón, 2010). Aunado a ello, es vital incorporar la comprensión y el análisis del contexto, con todas las variables históricas, políticas y socioculturales que 
implican la enseñanza y el aprendizaje del inglés. Es decir, considerando el punto de vista Freiriano, el papel de la formación del docente de inglés debe fundamentarse desde una visión sociocrítica que le empodere como agente de cambio de su entorno. Lo anterior, previendo que la lingüística meramente descriptiva e imperante sea convertida en una lingüística crítica aplicada (Chacón, 2010).

\section{Desarrollo profesional de docentes de inglés en Costa Rica}

Desde el 2010, bajo el Convenio Marco de Cooperación MEP-CONARE, en el nivel nacional, se estableció un plan de capacitación urgente a profesores en servicio del MEP. A partir de este acuerdo, las universidades estatales han intensificado el diseño y la implementación de cursos en áreas como métodos, técnicas, desarrollo de materiales y evaluación (Calderón y Mora, 2015). A pesar de los esfuerzos, los mecanismos de formación permanente continúan siendo insuficientes en el país, especialmente en la Región Brunca.

Además, en el 2018, como una respuesta de alerta ante las falencias atribuidas a la preparación del profesorado de inglés, el entonces presidente señaló como prioritaria la enseñanza de esta asignatura y, entre sus acciones, destaca un plan de capacitación para elevar la calidad de la enseñanza del idioma. Atendiendo ese llamado nacional, CONLA UNA, evento que congrega a dos poblaciones: el profesorado de inglés en servicio del MEP y el profesorado universitario, continúa siendo una contestación a la convocatoria para afianzar el desarrollo profesional de los docentes, promoviendo la excelencia del proceso de enseñanza-aprendizaje de dicha lengua.

\section{Antecedentes del proyecto CONLA UNA}

El proyecto Conferencia Internacional de Lingüística Aplicada (CONLA UNA) en la Región Brunca, gestado por la Universidad Nacional, campus Pérez Zeledón (UNA PZ) se diseñó e implementó desde la premisa de que las conferencias académicas y profesionales constituyen una actividad imprescindible en el quehacer universitario. El proyecto consiste en el fortalecimiento de la formación en servicio de docentes de inglés de la Zona Sur, a través de la ejecución y el desarrollo de cuatro conferencias de lingüística aplicada. Llevar a cabo estas conferencias, bajo la modalidad de proyecto desde la Universidad Nacional, surgió a raíz de la necesidad propia de educadores en áreas 
rurales de trasladarse hasta la capital del país para asistir a acontecimientos similares (Barrantes y Olivares, 2017).

Para el propósito de este documento, la formación en servicio se entiende como un componente del desarrollo docente, en el cual los educadores tienen un espacio de aprendizaje y retroalimentación paralelo a sus funciones de docencia. En cuatro diferentes ediciones, que iniciaron en el 2013, CONLA UNA ha sido una herramienta para dinamizar y mediar a través de los interaprendizajes y donde se ha utilizado la conversación como estrategia de aprendizaje continuo. Paralelamente, CONLA UNA se ha implementado con el interés de ofrecer espacios para aprender entre iguales, con un lenguaje dialógico, por medio de la participación de maestros de primaria, secundaria, educación técnica y educación superior.

Esta serie de conferencias ha ofrecido conversatorios, talleres, ponencias y plenarias, en las cuales se ha brindado propuestas metodológicas e investigativas de alto valor en el área de inglés. El aporte de este proyecto al fortalecimiento de los profesionales se ha visto reflejado en el proceso continuo de actualización e innovación en el campo de la enseñanza del inglés como lengua extranjera.

Aunado a dicha necesidad, se evidencia el compromiso del Consejo Nacional de Rectores, que, a través de la Oficina de Planificación de la Educación Superior en su Plan Nacional de la Educación Superior Universitaria Estatal 2016-2020, establece el requerimiento de reforzar la educación continua. Bajo el eje Pertinencia e Impacto, el Plan alude al papel que desempeña la educación superior estatal en la sociedad y a

la capacidad de las instituciones para responder en forma innovadora, a las demandas y necesidades del país y a las tendencias globales, desde diversas perspectivas, con diferentes instrumentos y modalidades. Involucra la docencia por todo el aporte de las personas graduadas; además al eje de Extensión y Acción Social con los proyectos que desarrolla, y a las acciones de educación continua. (CONARE, 2005, p. 102)

Así, el escrito, además, hace énfasis en la obligación de la UNA $\mathrm{PZ}$ de dar seguimiento a sus egresados. Establece como meta estratégica "fortalecer los vínculos recíprocos entre la universidad y sus egresados para promover la actualización cognoscitiva y la adaptación 
a circunstancias cambiantes" (CONARE, 2005, p. 100). De manera más específica, se compromete a "acentuar el seguimiento y comunicación de las universidades con sus egresados, e identificar sus requerimientos en educación continua" (CONARE, 2005, p. 100).

\section{La enseñanza de idiomas en la Región Brunca}

La carrera Bachillerato en la Enseñanza del Inglés inició en 1997, en el Campus Pérez Zeledón, y en el 2011, en el Campus Coto. Esta institución fue la primera, en la zona, en ofrecer la carrera en modalidad presencial. Desde el 2000 a la fecha, la institución gradúa, anualmente, profesionales que son recibidos en el mercado laboral, mayormente en instituciones de enseñanza. Debido al compromiso de la universidad por dar seguimiento a sus egresados, se realizó un análisis exploratorio sobre la oferta de espacios de formación profesional para docentes de inglés en la Zona Sur. Este análisis incluyó maestros de inglés en servicio, junto con autoridades de la UNA PZ, y reveló que la institución necesita fortalecer sus iniciativas de seguimiento a egresados del Bachillerato en la Enseñanza del Inglés, así como a profesores de la región que se encuentran laborando (Barrantes y Olivares, 2017).

A pesar de que las carreras Bachillerato en la Enseñanza del Inglés y Licenciatura en Lingüística Aplicada del inglés de este campus han graduado un gran número de expertos en esta área ${ }^{3}$, los mecanismos empleados para darle sostenibilidad y seguimiento a la excelencia de dichos profesionales se resume en las cuatro ediciones de CONLA UNA.

\section{Estrategia metodológica para la evaluación del proyecto}

La comisión organizadora del proyecto estableció como objetivo general para todas las ediciones: desarrollar una conferencia que promueva la formación en servicio con espacios de actualización, sobre cómo aplicar tanto prácticas como estrategias pedagógicas y de investigación, que fortalezcan el quehacer de la Lingüística Aplicada del inglés en la Región Brunca. Algunos de los objetivos específicos que se establecieron fueron: fortalecer el vínculo profesional entre la Universidad Nacional, otras universidades públicas y privadas y entes gubernamentales; promover la actualización y el uso de nuevas metodologías, estrategias e investigaciones en el campo de la lingüística

3 La base de datos de la Oficina de Registro informa de 293 graduandos en la carrera Bachillerato en la Enseñanza del Inglés y 17 en Licenciatura en Lingüística Aplicada del Inglés. 
aplicada, y analizar los resultados de la conferencia para fortalecer futuros eventos.

Como comisión responsable, las desarrolladoras del evento sistematizaron las acciones para la ejecución de todas las ediciones y redactaron informes finales durante los años 2013, 2015, 2017 y 2019 que fueron presentados a las autoridades competentes. Esos informes proveyeron la mayoría de los datos para esta evaluación.

La evaluación de proyectos constituye un procedimiento determinante en el desarrollo de prácticas académicas en la educación superior. A través de este proceso de valoración, se pueden examinar los elementos que intervienen en el proyecto, para así determinar su impacto, desempeño, viabilidad, riesgos, percepción externa e interna, oportunidades y capacidades. El procedimiento implica más que valorar, ya que provee mejores condiciones para la toma de futuras decisiones fundamentales.

Basados en los principios de evaluación de programas sugeridos por Fitzpatrick et al. (2011), esta evaluación tuvo como objetivo principal determinar el impacto de CONLA UNA como herramienta de desarrollo profesional, en la Sede Brunca. Para esto, se siguieron tres enfoques: evaluación orientada a programas, evaluación orientada a la toma de decisiones y evaluación orientada a los participantes.

El sector participante incluye los asistentes a las conferencias, los coordinadores de las subcomisiones del proyecto y las dos desarrolladoras principales. Los participantes son todos los profesores, los presentadores, los estudiantes-edecanes y los estudiantes universitarios que se apersonaron y fueron encuestados al final de cada edición de la conferencia. Los coordinadores pertenecían a las comisiones científica, de protocolo y eventos sociales, logística interna, logística externa, promoción y divulgación, y financiera. Su involucramiento a lo largo de los seis años fue constante, con algunas pequeñas variaciones. Finalmente, las desarrolladoras y evaluadores del proyecto intervinieron en sesiones de análisis, redactaron los informes y completaron una encuesta.

Se diseñaron tres instrumentos para la evaluación de las conferencias, uno dirigido a los participantes, otro a los presentadores y uno más a los coordinadores de las subcomisiones. El instrumento para participantes tiene seis partes: la actividad, ponencias, plenarias, logística interna, edecanes, ambiente físico. El instrumento para los presentadores consta de cinco partes: proceso de inscripción, la actividad, 
plenarias, logística interna, edecanes, ambiente físico. En su parte final, los dos instrumentos solicitan a los encuestados hacer sugerencias para mejorar la próxima edición. Estas herramientas se aplicaron al finalizar cada edición, en los años 2013, 2015, 2017 y 2019. Por último, el instrumento dirigido a los coordinadores utilizó el análisis sugerido para la evaluación orientada a la toma de decisiones, que recomiendan Fitzpatrick et al. (2011). Este incluye una orientación formativa, para la toma de decisiones, y otra sumativa, para la rendición de cuentas.

Además de los instrumentos, se analizaron documentos oficiales. A estos los constituyeron los informes finales y las formulaciones de proyectos del Sistema de Información Académica de la institución. Tales textos contemplan información longitudinal del diseño, planificación, desarrollo, rendición de cuentas y evaluación de cada una de las ediciones.

\section{Resultados de la evaluación}

Esta sección presenta los resultados de la evaluación desde tres enfoques: evaluación orientada a programas, evaluación orientada a la toma de decisiones y evaluación orientada a los participantes. Una de las finalidades de esta conferencia académica es proveer espacios de formación en servicio, que promuevan la actualización sobre cómo aplicar tanto prácticas como estrategias pedagógicas y de investigación, por lo que indagar con los participantes resulta clave para fortalecer las competencias personales y profesionales de los docentes, así como para proveer oportunidades que construyan e integren la comunidad.

\section{Evaluación orientada a programas}

Este enfoque de evaluación atiende los objetivos y propósitos del proyecto, así como la medida en que estos se cumplieron. El objetivo principal de CONLA UNA, durante todas sus ediciones, fue desarrollar una conferencia, durante tres días consecutivos, que promueva un espacio de actualización sobre cómo aplicar tanto prácticas como estrategias pedagógicas y de investigación, que fortalezcan el quehacer de la lingüística aplicada en la Región Brunca.

Primeramente, en las cuatro ediciones, la conferencia se extendió tres días consecutivos en el campus Pérez Zeledón, con una asistencia consistente durante esas fechas y una cantidad de participantes similar en todas las ediciones (ver tabla 1). De acuerdo con los datos recolectados por las desarrolladoras del proyecto, a lo largo del proceso de 
redacción de cada uno de los cuatro informes, se implementaron cinco espacios para participar: talleres, ponencias, conversatorios, plenarias y la técnica del carrusel. La modalidad de ponencias fue la más popular de todas (ver tabla 1), mientras que las demás modalidades siempre contaron con números muy bajos.

Los espacios dirigidos a la aplicación de prácticas pedagógicas fueron mayores que los de las prácticas investigativas. Es importante aclarar que todas las presentaciones en la modalidad de ponencias requerían un fuerte marco de investigación. Sin embargo, a pesar de ser indagaciones, estas se estructuraron de manera tal que su enfoque era el compartir prácticas efectivas en la enseñanza del inglés. Las presentaciones de prácticas investigativas representaron un número menor en todas las conferencias. Finalmente, entre los ejes temáticos (lingüística, enseñanza y aprendizaje de idiomas, TIC, cultura y literatura), la enseñanza y aprendizaje de idiomas constituyó el área de mayor interés para los participantes.

\section{Tabla 1}

Inventario de alcances del objetivo principal

\begin{tabular}{lllll}
\hline & $\begin{array}{c}\text { Primera } \\
\text { edición }\end{array}$ & $\begin{array}{c}\text { Segunda } \\
\text { edición }\end{array}$ & $\begin{array}{l}\text { Tercera } \\
\text { edición }\end{array}$ & $\begin{array}{l}\text { Cuarta } \\
\text { edición }\end{array}$ \\
\hline $\begin{array}{l}\text { Fecha de edición de la } \\
\text { conferencia }\end{array}$ & $\begin{array}{l}4,5 \text { y } 6 \\
\text { de febrero } \\
\text { del } 2013,29\end{array}$ & $\begin{array}{l}25,26 \\
\text { y } 30 \text { de } \\
\text { octubre } \\
\text { del } 2015\end{array}$ & $\begin{array}{l}\text { y 27 de } \\
\text { octubre } \\
\text { del } 2017\end{array}$ & $\begin{array}{l}\text { y 29 de } \\
\text { marzo del } \\
2019\end{array}$ \\
\hline Número de participantes & 160 & 114 & 112 & 84 \\
\hline Tipos de espacios & & & & \\
\hline Talleres & 5 & 7 & 13 & 9 \\
\hline Ponencias & 27 & 26 & 26 & 24 \\
\hline Conversatorios & 1 & - & 2 & - \\
\hline Plenarias & 4 & 3 & 5 & 6 \\
\hline Técnica del carrusel & - & - & 6 & 6 \\
\hline Total & 37 & 36 & 52 & 45 \\
\hline Cantidad de espacios que promovieron & & \\
\hline Estrategias pedagógicas & 26 & 26 & 46 & 29 \\
\hline $\begin{array}{l}\text { Estrategias de } \\
\text { investigación }\end{array}$ & 8 & 10 & 6 & 16 \\
\hline Total & 37 & 36 & 52 & 45 \\
\hline
\end{tabular}




\begin{tabular}{lllll}
\hline & $\begin{array}{c}\text { Primera } \\
\text { edición }\end{array}$ & $\begin{array}{c}\text { Segunda } \\
\text { edición }\end{array}$ & $\begin{array}{c}\text { Tercera } \\
\text { edición }\end{array}$ & $\begin{array}{c}\text { Cuarta } \\
\text { edición }\end{array}$ \\
\hline Áreas de lingüística aplicada & desarrolladas & & \\
\hline Lingüística & 1 & 2 & 1 & 4 \\
\hline $\begin{array}{l}\text { Enseñanza y aprendizaje } \\
\text { de idiomas }\end{array}$ & 26 & 27 & 33 & 25 \\
\hline TIC & 2 & 4 & 12 & 8 \\
\hline Cultura & 7 & 2 & 3 & 3 \\
\hline Literatura & 1 & 1 & 3 & 5 \\
\hline Total & 37 & 36 & 52 & 45 \\
\hline
\end{tabular}

Nota: Elaboración propia, con base en los informes de la comisión organizadora, 2013-2019.

Todos los coordinadores encuestados revelaron que el objetivo del proyecto fue alcanzado. Mencionaron que, a pesar de factores externos como desastres naturales, huelgas en el sector educativo, cambio de puesto de personas en las alianzas, entre otras cosas, se cumplió con el objetivo de ofrecer a la comunidad de profesionales en enseñanza del inglés un espacio de formación continua y actualización.

De igual forma, los participantes asistentes durante las cuatro ediciones fueron consultados sobre el grado en el que el objetivo del evento fue cumplido (ver figura 1). La información del objetivo fue divulgada en las páginas web que anunciaban y describían el evento. En esas mismas páginas, los participantes realizaban su inscripción. De manera muy positiva, los porcentajes de las cuatro ediciones demuestran el alto grado de cumplimiento de este objetivo. 


\section{Figura 1}

Grado de cumplimiento de los objetivos

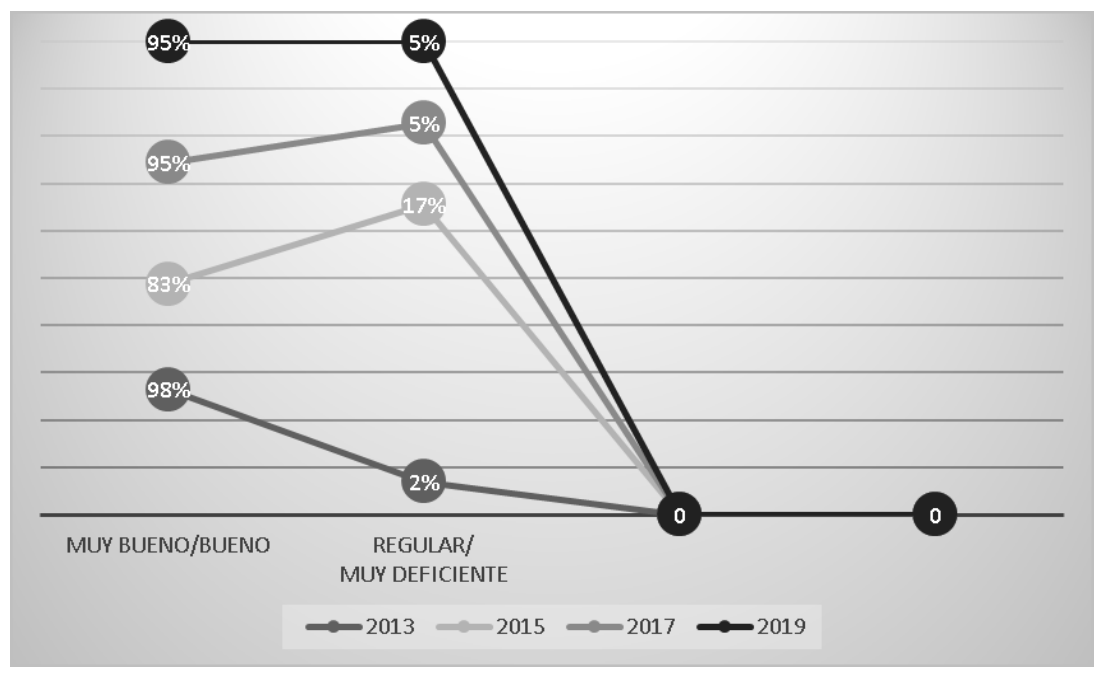

Nota: Los resultados se obtuvieron de 240 cuestionarios aplicados entre los años 2013, (99), 2015 (42), 2017 (78) y 2019 (21).

Así como se estableció en el Sistema de Información Académica de la Universidad Nacional, en el rubro de productos esperados, el proyecto se propuso construir alianzas estratégicas con organizaciones y empresas, así como formar y consolidar redes de cooperación. Los informes reportan uniones continuas con la Embajada de los Estados Unidos en Costa Rica y el Centro de Idiomas de la UNA (CI-UNA), las cuales brindaron un apoyo económico importante. El respaldo de la Embajada de los Estados Unidos en Costa Rica se concretó en el financiamiento de la participación de plenaristas internacionales durante las cuatro ediciones. El Ministerio de Educación Pública, por su parte, mostró su apertura para gestionar la intervención de sus docentes y de su asesor educativo. Diferentes empresas dejaron ver su soporte a través de patrocinios y facilitación de talleres (ver tabla 2). Las editoriales Oxford y Richmond financiaron y gestionaron talleres que fueron altamente evaluados por los participantes, gracias a su dinamismo y contenido. 


\section{Tabla 2}

Resumen de alianzas y colaboración

\begin{tabular}{|c|c|c|c|c|}
\hline & $\begin{array}{l}\text { Primera } \\
\text { edición }\end{array}$ & $\begin{array}{l}\text { Segunda } \\
\text { edición }\end{array}$ & $\begin{array}{l}\text { Tercera } \\
\text { edición }\end{array}$ & $\begin{array}{l}\text { Cuarta } \\
\text { edición }\end{array}$ \\
\hline & $\begin{array}{l}4,5 \text { y } 6 \text { de } \\
\text { febrero del } \\
2013\end{array}$ & $\begin{array}{l}28,29 \text { y } 30 \\
\text { de octubre del } \\
2015\end{array}$ & $\begin{array}{l}25,26 \text { y } 27 \\
\text { de octubre del } \\
2017\end{array}$ & $\begin{array}{l}27,28 \text { y } 29 \\
\text { de marzo del } \\
2019\end{array}$ \\
\hline Organizaciones & $\begin{array}{l}\text { Ministerio de } \\
\text { Educación } \\
\text { Pública } \\
\text { COLYPRO }\end{array}$ & $\begin{array}{l}\text { Ministerio de } \\
\text { Educación } \\
\text { Pública } \\
\text { COLYPRO }\end{array}$ & $\begin{array}{l}\text { Ministerio de } \\
\text { Educación } \\
\text { Pública }\end{array}$ & $\begin{array}{l}\text { Ministerio de } \\
\text { Educación } \\
\text { Pública } \\
\text { COLYPRO }\end{array}$ \\
\hline Emp & $\begin{array}{l}\text { Banco } \\
\text { Popular } \\
\text { ICAFE } \\
\text { Coopealianza } \\
\text { Oxford } \\
\text { University } \\
\text { Press } \\
\text { Librería La } \\
\text { Doce }\end{array}$ & $\begin{array}{l}\text { Banco } \\
\text { Popular } \\
\text { Oxford } \\
\text { University } \\
\text { Press } \\
\text { Richmond } \\
\text { Publishing } \\
\text { Samsung } \\
\text { Corporation } \\
\text { Librería La } \\
\text { Doce }\end{array}$ & $\begin{array}{l}\text { Banco } \\
\text { Popular } \\
\text { Oxford } \\
\text { University } \\
\text { Press } \\
\text { Sid de Costa } \\
\text { Rica } \\
\text { Librería } 506 \\
\text { Coopeagri } \\
\text { R. L. } \\
\text { Librería La } \\
\text { Doce }\end{array}$ & $\begin{array}{l}\text { Oxford } \\
\text { University } \\
\text { Press } \\
\text { Richmond } \\
\text { Publishing } \\
\text { Sid de Costa } \\
\text { Rica } \\
\text { Librería } 506 \\
\text { Coopeagri } \\
\text { R. L. } \\
\text { Kölbi }\end{array}$ \\
\hline $\begin{array}{l}\text { Redes de } \\
\text { cooperación }\end{array}$ & $\begin{array}{l}\text { CI-UNA } \\
\text { Embajada de } \\
\text { los Estados } \\
\text { Unidos }\end{array}$ & $\begin{array}{l}\text { CI-UNA } \\
\text { Embajada de } \\
\text { los Estados } \\
\text { Unidos }\end{array}$ & $\begin{array}{l}\text { CI-UNA } \\
\text { Embajada de } \\
\text { los Estados } \\
\text { Unidos }\end{array}$ & $\begin{array}{l}\text { CI-UNA } \\
\text { Embajada de } \\
\text { los Estados } \\
\text { Unidos }\end{array}$ \\
\hline
\end{tabular}

Nota: Elaboración propia, con base en los informes de la comisión organizadora, 2013-2019.

\section{Evaluación orientada a la toma de decisiones}

Este enfoque de evaluación está dirigido a la gestión informada para la toma de decisiones asertivas. Se fundamenta en que la información evaluativa es una parte esencial para la buena toma de decisiones. Así, el evaluador puede ser más efectivo al servir a los administradores, gerentes, formuladores de políticas, juntas, profesionales y otros que necesitan información de calidad. Debido a sus funciones como gestores de procesos administrativos y de logística, los coordinadores de las subcomisiones fueron los informantes que proveyeron los datos para esta evaluación. Siguiendo las recomendaciones de Fitzpatrick et al. 
(2011), el instrumento completado incluyó una orientación formativa y sumativa para la toma de decisiones y la rendición de cuentas.

El primer punto del cuestionario por analizar incluyó el input, en el que se cuestionó la modalidad de conferencia de CONLA UNA como una elección asertiva de estrategia para el desarrollo profesional de profesores de inglés de la Zona Sur. Los encuestados respondieron que, conforme han pasado los años, han podido divisar dos tipos de participantes en el CONLA UNA. Por un lado, los docentes del Ministerio de Educación Pública (primaria y secundaria) prefieren presentaciones prácticas y dinámicas como talleres o el carrusel. Por otro lado, los profesores universitarios encuentran de mayor provecho las charlas donde se presentan datos y resultados de investigación. Este último grupo de participantes son los que representan la mayoría de los presentadores. Ello ha hecho difícil atender ambas demandas y seguir salvaguardando la naturaleza original del CONLA. Es a raíz de este conflicto que uno de los coordinadores sugirió cambiar la modalidad de conferencia por simposio o seminario, en el cual más expertos, menos actividades y menos ponentes pudiesen dar resultados mayormente efectivos para la agrupación de educadores universitarios.

Con respecto a los elementos de proceso y producto, los participantes enumeraron las actividades que resultaron exitosas, las que necesitan fortalecerse, las que deben ser replicadas por su efectividad y las que es preciso eliminar. Específicamente, la dinámica más exitosa fue la coordinación entre comisiones, por parte de la comisión organizadora, con un efectivo medio de comunicación (aplicación WhatsApp) y su cumplimiento de la línea de tiempo de trabajo propuesto. Mencionaron la disposición y voluntad de trabajo demostrada, aun cuando no hay jornada de tiempo asignada, y todo se elaboró con tiempo ad honorem. Los coordinadores también señalaron como triunfantes la formalidad y el orden en la ejecución de los eventos protocolarios y de esparcimiento; la coordinación con diferentes instancias para divulgar información sobre las conferencias; el acompañamiento a los profesores conferencistas, y la innovación en la tercera edición de la modalidad del carrusel. Debido a su éxito, para la última edición se requirió un espacio más amplio.

En el mismo análisis, se registraron las actividades protocolarias, invitación de pasantes internacionales, involucramiento de estudiantes asistentes como edecanes, la técnica del carrusel, reuniones y 
comunicación a través de WhatsApp con la comisión organizadora central, al igual que la distribución de símbolos externos, como iniciativas que deben ser repetidas en futuras ediciones. Entre las dinámicas por fortalecer están: ofrecer mayor variedad de eventos artísticos (comisión de protocolo); reforzar el apoyo de medios de comunicación internos y externos; mejorar la estrategia de divulgación en plataformas internacionales (comisión de divulgación); robustecer el vínculo con el Ministerio de Educación (comisión organizadora); incluir el elemento tecnológico para los procesos de inscripción; vigorizar el seguimiento y la evaluación (comisión organizadora); crear una comisión a la que se le asigne solamente la emisión de certificados (comisión organizadora); comunicar a los asistentes el rol y las responsabilidades de los edecanes (comisión logística interna). Los coordinadores expresaron su interés por crear una estrategia que les permita participar de las presentaciones, sin que esto comprometa sus asignaciones, ya que el organizar algunas actividades les impidió asistir a la mayoría de aquellas. De manera explícita, la mayoría de los coordinadores manifestó la necesidad de fortalecer la táctica para captar los encargados de las comisiones. Ellos insistieron en que su intervención debería ser una iniciativa o invitación, y no una imposición, para crear grupos de trabajo más afines y lograr las responsabilidades de cada comisión no se recarguen en quien las dirige.

Finalmente, según los coordinadores, las actividades que deben ser eliminadas son ofrecer transporte a los conferencistas, ya que se considera que estos deben llegar por sus propios medios; no imponer la asignación a los organizadores, sin una previa conversación; no enfatizar la contribución de todo tipo de patrocinadores, especialmente de aquellos que no están relacionados con los objetivos del evento. También, mencionaron la posibilidad de reducir la conferencia a uno o dos días.

\section{Evaluación orientada a los participantes}

En este apartado, se presentan y analizan los resultados de las impresiones de 240 asistentes a los eventos. Los participantes de este proyecto los constituyen los asistentes en modalidad de audiencia, los presentadores de las diferentes modalidades y los estudiantes-edecanes. Todos ellos fueron invitados a completar una encuesta al final de cada edición. Sus respuestas fueron sistematizadas e incluidas en los cuatro reportes finales (ver tabla 3.) La primera parte de la encuesta contempla los enunciados de la organización de la actividad en general. Aquí, los 
porcentajes demuestran el alto nivel de agrado con respecto al cumplimiento de los objetivos y de las actividades programadas, así como de la calidad y disponibilidad de la información. En el 2015, se identifica un declive, pues aparecen porcentajes más altos de insatisfacción, sin embargo, para las dos ediciones siguientes, ese rubro demuestra altos niveles nuevamente.

De la pregunta dos sobre ponencias y plenarias, los participantes indicaron que la calidad académica de los temas presentados fue de alta satisfacción. Los porcentajes elevados que se otorgaron a este enunciado revelan el nivel favorable de dominio temático de los ponentes, su capacidad para despertar interés y tanto la calidad del tema como la disponibilidad para responder preguntas y aclarar dudas. Algunos de los comentarios de los participantes indican un interés colectivo por que las conferencias tengan un alcance más práctico y apegado a aspectos meramente de praxis pedagógica. Entre sus reflexiones, se registran "me hubiese gustado que se presentaran más actividades", "me gustaría que los talleres sean más pertinentes a la labor docente", "por favor incluir más talleres", "que más personas expongan técnicas prácticas" y "por favor no incluir tantas investigaciones." La modalidad del carrusel registró un gran interés en esas acotaciones.

Ante la interrogante sobre logística interna, los encuestados comentaron que el proceso de inscripción, el programa del evento y la organización de los espacios en donde se realizaron las presentaciones fueron óptimos y evaluados de manera positiva. Para los años 2017 y 2019, se registran porcentajes menos positivos, sin embargo, de manera general, los porcentajes altos se mantuvieron. Algunos comentarios constantes incluyeron recomendaciones sobre los refrigerios y la necesidad de proveer alimentación oportuna, ya que muchos de los participantes se desplazaron de lugares lejanos. También, se hizo referencia a lo importante de cumplir con detalle el programa establecido, pues se registraron inconformidades cuando se hicieron cambios sin previo aviso. Una acotación contradictoria fue la de duplicar presentaciones. Algunos participantes criticaron que una misma presentación se realizara en diferentes ocasiones, mientras otros afirmaron que esta estrategia les permitió asistir a todas las exposiciones. 


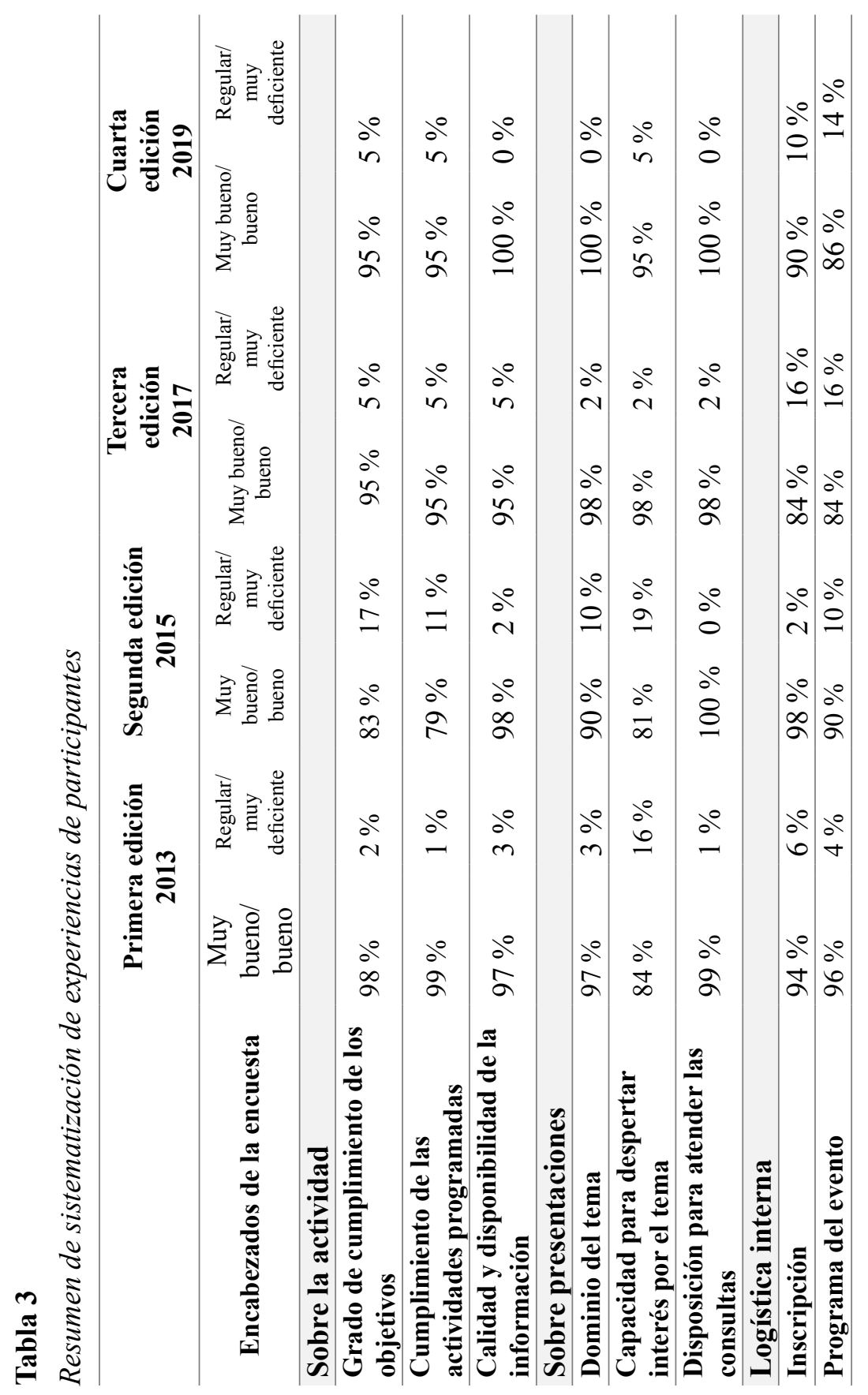




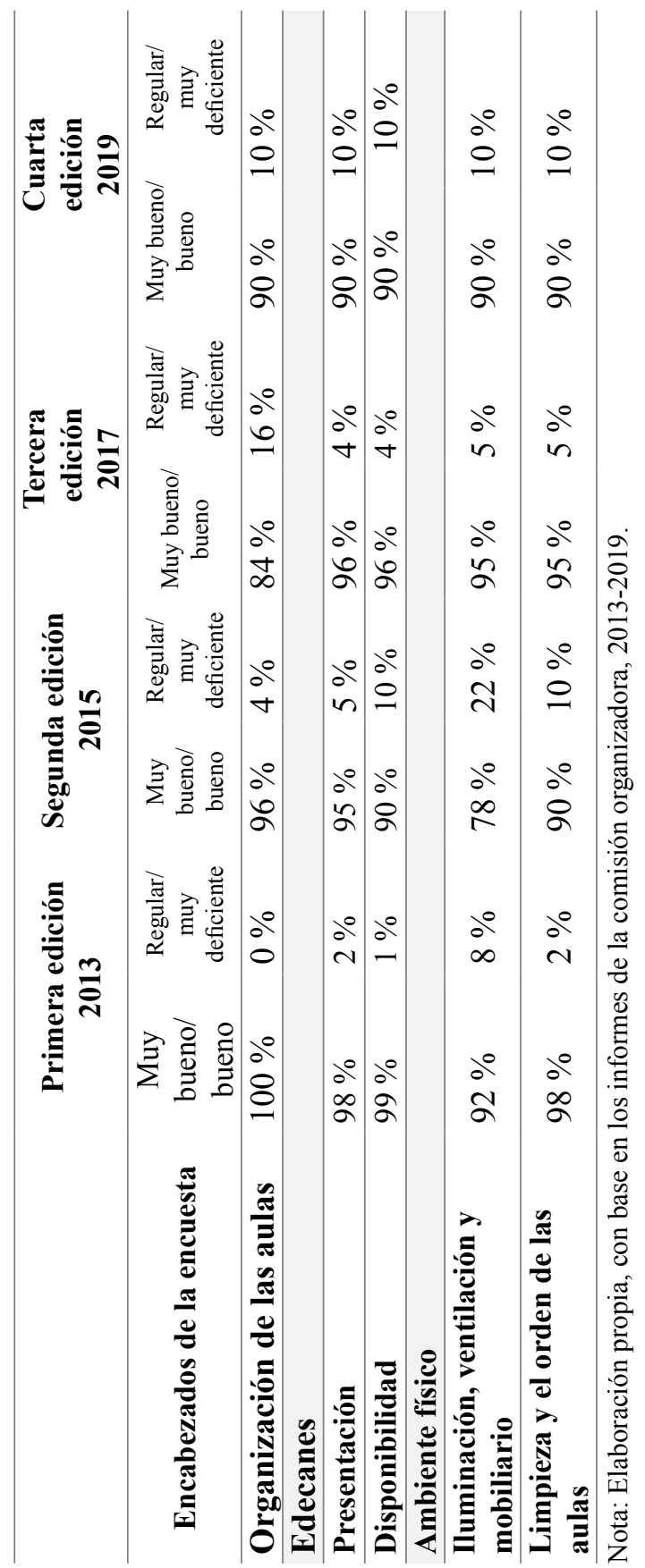


De la pregunta sobre el papel de los edecanes, se distingue entre la presentación y disponibilidad de estos para responder consultas y brindar ayuda. Aun cuando se registra un declive para el 2019, los porcentajes demostraron una evaluación alta de su desempeño. Finalmente, los asistentes opinaron que aspectos del espacio físico como iluminación, ventilación, mobiliario, limpieza y orden necesitaban ser mejorados en la edición del 2015. Un aspecto importante por tomar en cuenta es la cantidad de participantes que evaluaron las ediciones, puesto que a lo largo de los años menos cuestionarios fueron aplicados y analizados.

\section{Discusión}

El objetivo principal de esta evaluación fue analizar el impacto positivo de la modalidad de conferencia como herramienta de desarrollo profesional en la Zona Sur. Para esto, se examinó su impacto, desempeño y viabilidad (oportunidades y riesgos). La discusión de su efecto la constituye la percepción externa (participantes-asistentes) e interna (coordinadores y desarrolladoras).

Con respecto a su impacto, el proyecto registra un total de 524 inscripciones. Profesionales en el área de la enseñanza del inglés de educación primaria, secundaria y universitaria participaron y compartieron sus experiencias, las cuales narran con agradecimiento, gracias al espacio para compartir con colegas e intercambiar experiencias pedagógicas. Por esta vía, más que renovar el conocimiento, se plantean nuevas soluciones a través del diálogo. Existe la necesidad de trascender las sociedades del conocimiento o de la información y evolucionar hacia comunidades de aprendizaje en red. La conferencia como modalidad de desarrollo profesional ofrece espacios para la conexión presencial entre docentes, que puede catapultar relaciones más fuertes y sistemáticas.

Como lo explica Sorokina (2015), la conferencia académica remite un discurso expositivo oral en el que se distinguen dos metas: a) divulgar y difundir las investigaciones presentadas por el conferencista; b) la intervención reflexiva por parte del asistente-participante durante el desarrollo del evento. Lo popular de la técnica del carrusel se justifica con la idea de la misma Sorokina (2015), quien califica el espacio de charla o conversación como más amigable, al ser dialógico por naturaleza. 
Ahora bien, Mingorance y Estaranz (2009) recalcan la función universitaria en términos de promover y concretar una comunidad de aprendizaje. Las conferencias en cuestión probaron ser un espacio de conexión y diálogo entre profesionales y organizaciones. Se cree que las universidades son el centro de la revolución del aprendizaje; tienen la misión y la responsabilidad de promover acciones orientadas a la comunidad, a través de redes de gente comprometida. Las cuatro ediciones, en un lapso de seis años, demuestran el aporte a la colectividad profesional hacia la cual fueron dirigidas.

Por otra parte, el desempeño de las conferencias fue marcado por la participación comprometida de los coordinadores de todas las comisiones. Su involucramiento y evaluación sacan a la luz la clasificación de dos diferentes grupos de asistentes que discrepan en sus intereses. Están los profesores de inglés de primaria y secundaria que laboran para el Ministerio de Educación, quienes persiguen espacios de práctica pedagógica que les permitan aplicar los conocimientos compartidos en sus clases. En cambio, los docentes universitarios buscan en la conferencia un compartir del saber más enfocado en investigación de lingüística aplicada que les conceda crecer en conocimiento. Esta discrepancia abre espacio para considerar lo urgente de realizar cambios estratégicos en la organización, los cuales satisfagan ambas necesidades, sin comprometer el objetivo principal del proyecto. Como lo explican Fitzpatrick et al. (2011), la información que proveen, en este caso los coordinadores, posibilita que cada componente del proyecto reciba apoyo, mientras este se desarrolla, crece y cambia.

Entre las oportunidades que se registran, las alianzas con la Embajada de los Estados Unidos en Costa Rica y las editoriales fortalecen la calidad y la orientación temática. A pesar de que los convenios se hicieron de forma temporal, previos a ejecutar cada edición, el involucramiento ha tenido un historial permanente durante los cuatro eventos. Además, su impacto positivo apela por continuar su participación. Estas uniones son recursos que se pueden aprovechar o utilizar para hacer posible el logro de los futuros objetivos.

Los principales riesgos de las actividades contemplan el elevado gasto económico, la disminución del número de participantes y la discrepancia de intereses de estos. Se documentó que la ayuda financiera interna y externa es necesaria para el sostenimiento de futuras ediciones. De la primera a la última edición, el número de participantes 
disminuyó en un $50 \%$. Tal mengua puede ser el resultado de los diferentes propósitos de las poblaciones participantes. Como se aludió, docentes de primaria y secundaria buscan un espacio interactivo, el cual les permita recolectar material y prácticas efectivas que puedan replicar o experimentar en sus clases. En otro sector, la mayoría de ponentes universitarios compartieron su enfoque investigativo, hecho esperado y demandado en un ambiente académico. Esta discrepancia resulta en un conflicto de intereses que necesita ser dirigido de modo estratégico.

\section{Recomendaciones para el desarrollo de conferencias}

Tanto participantes como coordinadores y evaluadores describieron una serie de sugerencias para la ejecución de distintos procesos. Los siguientes son consejos para el desarrollo de la conferencia, por parte de los participantes:

- Mantener excelente organización y cumplimiento de actividades.

- Habilitar el registro electrónico.

- Evitar que la fecha sea en octubre (por las lluvias).

- $\quad$ Mantener el lounge para presentadores.

- $\quad$ Fortalecer la divulgación del evento.

- Incluir material práctico en las ponencias.

- Agregar más talleres.

- Exponer más técnicas prácticas.

- Incorporar más expertos internacionales.

- $\quad$ Proveer un espacio más amplio para el carrusel.

Los coordinadores por su parte recomendaron:

- Transicionar a una modalidad de simposio o seminario (más expertos, menos actividades, menos ponentes).

- No tratar de abarcar una población que no siempre ha demostrado interés genuino en el aprovechamiento de la oportunidad formativa (profesores del Ministerio de Educación), sino, más bien, enfocarse en una participación voluntaria y que dé mayor énfasis a la intervención experta.

- Convertir el campus donde se desarrolla la conferencia en itinerante, cada dos años en un campus diferente. Esto con el fin de abarcar más población de los otros cantones de la Región Brunca. 
- $\quad$ Reanudar la redacción de una memoria indexada.

- Reestructurar el ingreso económico. El evento debe sostenerse por sí solo y no depender de los fondos internos de la universidad ni de patrocinadores. Para esto, se requiere incrementar la cuota de inscripción.

- Postular la participación de los estudiantes edecanes, en la cual su rendimiento académico sea el criterio principal para su escogencia.

- $\quad$ Diversificar el papel de los estudiantes edecanes.

Finalmente, la comisión organizadora central del proyecto aconseja:

- Incorporar la técnica del carrusel.

- Potenciar el trabajo en equipo de los coordinadores.

- Mantener la comunicación abierta y constante con los coordinadores.

- Mantener la rigurosidad de la comisión científica en el proceso de escogencia de ponencias.

- Fortalecer la cooperación de entes externos, a través de una coordinación sólida, un diálogo asertivo y un apoyo comprometido.

- Consolidar la divulgación del evento en plataformas internacionales.

- Fortalecer los mecanismos cooperativos con el Ministerio de Educación.

- Mantener y consolidar las alianzas con la Embajada de los Estados Unidos y CI-UNA.

\section{Conclusiones}

Tomando como base epistemológica que el conocimiento se construye cognitivamente, a partir de la experiencia y la interacción del individuo con los demás y el entorno (Lincoln et al., 2018), la evaluación de proyectos como estrategia de mejoramiento educativo permite documentar las voces de los participantes. Estos son quienes, por medio de sus múltiples realidades e interpretaciones, proveen los parámetros de referencia y hallazgos que instan el mejoramiento de prácticas educativas.

Aun cuando esta evaluación busca impactar un proyecto en particular en un contexto específico, sus resultados informan acciones 
exitosas que benefician proyectos de desarrollo profesional similares. Esta valoración contribuye en diseminar datos que, a su vez, aportan insumos y posibilitan tomar decisiones bien fundamentadas y asertivas para futuras ediciones de la conferencia. Además, se provee información precisa a entidades académicas y administrativas interesadas que ostentan responsabilidad en el proyecto.

El papel emancipador que otorga la universidad al individuo cuando difunde conocimiento es vital para procurar la transformación en esta sociedad moderna. Con la retroalimentación aportada por participantes, coordinadores y desarrolladoras, se pretende proponer mejoras que contribuyan a la gestión de eventos similares, con un énfasis en el rol de la enseñanza del inglés como lengua extranjera en la Región Brunca.

Esta evaluación contribuye a la propuesta de seguir desarrollando conferencias académicas con estrategias de gestión que benefician a los participantes, presentadores y ejecutores; así se prepara a los futuros profesores, docentes en servicio e investigadores de esta carrera y se favorece la necesidad de interacción del maestro quien aprende en colaboración con otros. Al decir de Maturana (1997), el ser humano tiene la virtud de la adecuación para atender y dar respuesta en diferentes direcciones y grados de asistencia, según lo requiera su entorno. Las redes de relaciones colaborativas, como las conferencias académicas dirigidas a los docentes, promueven la retroacción y la humanización de los vínculos sociales (Galán, 2017).

\section{Referencias}

Algren, M., Dwyer, E., Eggington, B. y Witt, B. (2011). So, you agreed to chair a conference! En C. Coombe, M. L. McClosky, L. S y J. Anderson (Eds.), Leadership in English Teaching and Learning (pp. 102-113). The University of Michigan.

Barrantes, L. y Olivares, G. (2017). Fortalecimiento del liderazgo pedagógico en docentes de inglés en servicio a través de Redes de Educación Continua (REEDUCO) Sede Regional Brunca. Revista Electrónica Gestión de la Educación, 7(1), 17-36. https:// revistas.ucr.ac.cr/index.php/gestedu/article/view/27565

Calderón, R. y Mora, Y. (2015). Desarrollo profesional del docente de inglés en el área de evaluación de los aprendizajes. Revista 
Electrónica Actualidades Investigativas en Educación, 15(2), 1-22. https://doi.org/10.15517/aie.v15i2.18951

Chacón, C. T. (2010). Acento e identidad profesional en la formación del docente de inglés: Una perspectiva crítica. Paradigma, 31(2), 25-36. Consejo Nacional de Rectores (CONARE). (2015). Plan Nacional de la Educación Superior Universitaria Estatal 2016-2020. OPES.

Fitzpatrick, J., Sanders, J. y Worthen, B. (2011). Program evaluation: Alternative approaches and practical guidelines. Pearson.

Galán, B. (2017). Estrategias de acompañamiento pedagógico para el desarrollo profesional docente. Revista Caribeña de Investigación Educativa, 1(1), 34-52.

Guba, E. G. y Lincoln, Y. S. (2005). Pragmatic controversies, contradictions, and emerging confluences. En N. Y. Denzin y Y. S. Lincoln (Eds.), The SAGE Handbook of Qualitative Research (pp. 191215). Sage Publications.

Imbernón, F. y Canto, P. J. (2013). La formación y el desarrollo profesional del profesorado en España y Latinoamérica. Sinéctica Revista Electrónica de Educación, 41, 1-13. http://www.scielo.org.mx/ scielo.php?script $=$ sci_arttext\&pid $=$ S1665-109X2013000200010

Lincoln, Y. S., Lynham, S. A. y Guba, E. G. (2018). Pragmatic controversies, contradictions, and emerging confluences. Revisited. En N. Y. Denzin y Y. S. Lincoln (Eds.), The SAGE Handbook of Qualitative Research (pp. 97-150). Sage Publications.

Maturana, R. H. (1997). La realidad: ¿Objetiva o construida? Anthropos.UI.ITESO.

Mingorance, P. y Estebaranz, A. (2009). Construyendo la comunidad que aprende: La vinculación efectiva entre la escuela y la comunidad. Revista Fuentes, 9, 179-199.

Ruíz-Díaz, A., Roque-Doval, Y. y Rodríguez-Ruíz, M. (2017). Acciones de superación profesional para potenciar la competencia en comunicación de resultados científicos. Revista Electrónica Educare, 21(2), 1-23. https://doi.org/10.15359/ree.21-2.2

Sorokina, T. (2015). Género conferencial desde la semiótica compleja. Revista Iberoamericana de las Ciencias Sociales y Humanísticas, 4(8), 180-192. https://www.redalyc.org/articulo. oa? $\mathrm{id}=503950656011$

Wolfram, W. y Schilling-Este, N. (2006). American English. Blackwell Publishing. 